\title{
Gobernanza y participación social en la gestión del agua en la microcuenca El Cangrejo, en el municipio de Autlán de Navarro, Jalisco, México
}

\section{Governance and social participation in the management of water in El Cangrejo micro- basin, municipality of Autlán de Navarro, Jalisco, Mexico}

\author{
Aída Alejandra Guerrero-de León \\ Peter R.W. Gerritsen \\ Luis Manuel Martínez-Rivera \\ Silvia Salcido-Ruíz \\ Demetrio Meza-Rodríguez \\ Humberto Rafael Bustos-Santana*
}

\begin{abstract}
In this work the processes of water governance are analyzed from the intervention of the different social actors and institutional mechanisms developed for making decisions on the use and exploitation of water. The study was developed in the territory of El Cangrejo micro-basin, municipality of Autlan de Navarro, Jalisco. The theoreticalmethodological approach proposed by Hufty (2004), called analytic framework of governance $(A F G)$, was used.
\end{abstract}

Keywords: governance; nodal points; actors; micro-basin; sustainability; governance processes.

\section{Resumen}

En este trabajo se analizan los procesos de gobernanza del agua, a partir de la intervención de los diferentes actores sociales y los mecanismos institucionales desarrollados para la toma de decisiones del uso y aprovechamiento de agua. El estudio se desarrolló en territorio de la microcuenca El Cangrejo, municipio de Autlán de Navarro, Jalisco. Se utilizó el enfoque teórico-metodológico propuesto por Hufty (2004) denominado marco analítico de la gobernanza (MAG).

Palabras clave: gobernanza, gestión, puntos nodales, actores, microcuenca, sustentabilidad, procesos de gobernanza.

*Universidad de Guadalajara, México. Correos-e: guerreroleon40@hotmail.com, petergerritsen@ cucsur.udg.mx, lmartinez@cucsur.udg.mx, silesad@yahoo.com.mx, meza_rodriguezdemetrio@hotmail.com, hbustos1@yahoo.com.mx, humberto.bustos@cucsur.udg.mx. 


\section{Introducción}

El agua constituye una necesidad primordial para el desarrollo integral de las poblaciones humanas, sin embargo ya no es un recurso abundante y disponible en cantidad y calidad (UNDP, 2006). En México la mayor demanda de agua es para uso agrícola (88\%), seguida del uso industrial (7\%) y municipal (5\%), sin considerar la generación de energía; por una parte, esto ha ocasionado la sobreexplotación, y por otra, la contaminación de nuestros recursos hídricos (CNA, 2008). Por tanto, los gobiernos locales y los usuarios enfrentan un enorme reto para diseñar e implementar nuevos modelos de gestión integral de cuencas que aseguren la calidad y el abasto del agua a sus comunidades y generaciones futuras (UNDP, 2006; Barkin, 2007; unEsCo, 2009).

Actualmente diversos autores coinciden en que la participación ciudadana en los procesos de toma de decisiones contribuye a una mejor gestión del agua, y se considera una estrategia para lograr el desarrollo sustentable en el ámbito local (Ostrom, 1990; Córdova et al., 2006; Delgado et al., 2007; Galvin y Haller, 2008). A partir de la reunión de las Naciones Unidas para el Desarrollo y Medio Ambiente realizado en Río de Janeiro, Brasil, en 1992, los gobiernos de diversos países incluyen en sus políticas hídricas la estrategia del manejo integrado de los recursos hídricos (MIRH o IWRM, por sus siglas en inglés). ${ }^{1}$ Como consecuencia de estas nuevas estrategias y principios planteados en los foros mundiales del agua, en 1996 se formó la Global Water Partnership (GWP) para brindar las herramientas y la capacitación necesarias para implementar estas nuevas políticas.

El manejo integral de los recursos hídricos expone la necesidad de procesos intensos de descentralización y la integración de las poblaciones locales en el manejo, administración y aprovechamiento de sus recursos. En este contexto se plantea el tema de la gobernanza del agua como la clave para lograr la sustentabilidad ambiental (Rogers, 2002; Iza y Rovere, 2006; GWP, 2009). Así, la gobernanza se entiende como el conjunto de interacciones entre actores públicos y privados orientados a resolver sus problemas sociales para crear oportunidades en un marco normativo (Kooiman, 2003).

\footnotetext{
${ }^{1}$ Integrated Water Resources Management es resultado de acuerdos del Segundo Foro Mundial realizado en La Haya 2000. Los principios de IWRM se sustentan en los cuatro principios de Dublín y de Río de Janeiro, 1992: 1) el agua dulce es un recurso vulnerable y finito, esencial para mantener la vida, el desarrollo y el medio ambiente; 2) el desarrollo y manejo del agua se debe basar en un enfoque participativo, involucrando a usuarios, planificadores y realizadores de política a todo nivel; 3) la mujer juega un papel central en la provisión, manejo y protección del agua; y 4) el agua posee un valor económico en todos sus usos competitivos y se debiera reconocer como un bien económico.
} 
Se vuelve entonces prioritario entender cuáles son estos procesos o sucesos, es decir, los conflictos, acuerdos, normas e interacciones que se desarrollan en la toma de decisiones en un territorio delimitado por un sistema natural como la cuenca hídrica. Por tanto, las acciones que se realicen en una determinada parte de la cuenca tienen que ver con la calidad y cantidad del agua en otra parte de la misma (Dourojeanni y Jauravlev, 2001).

En México, en el marco del establecimiento de políticas internacionales ambientales, en 1992 surge la Ley de Aguas Nacionales (LAN) y con ella una serie de reformas en 2004. La LAN establece implementar procesos de descentralización y privatización de los recursos hídricos. Además esta ley propone la gestión integral en cuencas hídricas por medio de instituciones reconocidas como consejos de cuencas, donde se proponen espacios de participación ciudadana para la toma de decisiones. En México, aunque se han hecho esfuerzos por seguir con estos nuevos modelos de gestión del agua, aún es limitada la inclusión de todos los usuarios en los consejos de cuencas (Castro et al., 2004; Cotler, 2004).

Por tanto, el presente trabajo aborda el tema de la gobernanza del agua en el ámbito local, con el fin de entender cuáles son los principales conflictos por el uso del agua y cómo se desarrollan los procesos de toma de decisiones y acuerdos para solucionar sus problemas; asimismo se identificó cómo se articulan las políticas hídricas en la microcuenca. Por estas razones se seleccionó como caso de estudio la microcuenca El Cangrejo, que pertenece a la cuenca del río Ayuquila, de gran importancia para el desarrollo agrícola y por su riqueza de biodiversidad en la región costa sur del estado de Jalisco (Martínez et al., 2002; Gerritsen et al., 2005). El análisis de los procesos de gobernanza se realizó con la metodología propuesta por Hufty (2004) descrita como marco analítico de gobernanza (MAG), que consiste en identificar cinco categorías de análisis: problema, puntos nodales, actores, normas y procesos, método que describimos más adelante. A continuación exponemos los conceptos básicos para comprender mejor el tema de estudio.

\section{Marco teórico}

\subsection{Gobernanza y gobernanza del agua}

El término gobernanza tiene diferentes conceptualizaciones, dependiendo de quién y cómo se utilice. Por su origen etimológico es un término antiguo, del latín gubernare y del griego kybernân o kubernetes, que significa el que tiene el control de algo (Oliveira, 2002). En la actualidad gobernanza se confunde con el término gobernabilidad, entendido como 
"la capacidad de un gobierno para formular e implementar decisiones públicas" (Prats, 2003: 249). En cambio, gobernanza se puede describir, de acuerdo con sus aplicaciones, como un sinónimo de gobierno (un sentido explícitamente jerárquico), un marco normativo (una herramienta de medición de la capacidad del gobierno en los países, como propone el Banco Mundial) y como un marco analítico para los sistemas de coordinación no jerárquicos (corrientes teóricas nuevas de gobernanza global y moderna) (Aguilar, 2006).

Las corrientes teóricas relacionadas con el nuevo concepto de gobernanza, lo definen como el total de interacciones entre actores públicos y privados, para resolver problemas y crear oportunidades, entendiendo la participación de las instituciones formales o informales en un marco normativo, más como sistemas de redes entre actores y no sólo como una estructura jerarquizada en la toma de decisiones (Rhodes, 1997; Mayntz, 2001; Kooiman, 2003; Prats, 2003; Hufty, 2004). En este enfoque, Hufty (2004) propone un marco analítico de gobernanza (MAG) como una herramienta de análisis que propone identificar el conjunto de procesos (sucesión de estados), formales e informales, para solucionar conflictos sociales en un determinado tiempo y espacio. Este enfoque se utilizó para el desarrollo de este trabajo y se describe más adelante.

Los paradigmas actuales de la gestión del agua, ante los grandes problemas del agotamiento y deterioro de los recursos hídricos, proponen nuevos modelos de manejo que se relacionan precisamente con el concepto de gobernanza moderna (Rhodes, 1997; Kooiman, 2003), entendida desde los nuevos modelos de gestión pública (Aguilar, 2006). Esto implica cambiar los modelos actuales de planificación hídrica basados en la oferta y el desarrollo tecnológico, así como tener un enfoque de las teorías de la complejidad y la incertidumbre (Simonovic, 2000). Es decir, diseñar modelos apropiados de manejo frente a los cambios globales, los conflictos sociales y las instituciones. El reto de investigadores, planificadores y gobiernos ahora se deben centrar en analizar con qué valores y normas se debe gestionar el agua, planteando las diferencias de su distribución como un bien económico o un bien común en términos de desarrollo sustentable (Ostrom, 1990; Dourojeanni y Jauravlev, 2001; Rogers, 2002; Toledo, 2002).

Los esfuerzos de las instituciones internacionales por revertir la crisis del agua se establecieron en las conferencias de Dublín y de Río de Janeiro en 1992, organizadas por Naciones Unidas (ONU). En estos foros mundiales se plantearon agendas ambientales para aprovechar de forma adecuada el agua en términos de desarrollo sustentable, que después se tradujeron en los Objetivos del Milenio (PNUMA, 2000). Actualmente la agenda política ambiental promueve una nueva estrategia denominada 
gobernanza efectiva del agua (GWP, 2003: 1), que se refiere a establecer sistemas administrativos y sociopolíticos eficientes, además propone incluir los principios de manejo integral de los recurso hídricos (MIRH).

El término gobernanza eficaz es tema de debate, porque algunos investigadores consideran que en el contexto de sociedades complejas cambiantes es difícil establecer un calificativo (Ostrom, 1990). Más bien, la gobernanza del agua se debe entender como un proceso en el cual existen fallas u omisiones que pueden provocar que los conflictos no se solucionen. En estos procesos de gestión del agua se deben incluir mecanismos, procesos y espacios donde los ciudadanos participen y puedan expresar sus intereses, ejerzan sus derechos y donde se resuelvan sus diferencias. Estos procesos deben expresarse mediante marcos políticos, jurídicos, estrategias y planes de acción (Iza y Aguilar, 2006).

La gobernanza del agua se puede entender en el ámbito de una cuenca hídrica. En términos hidrológicos, la cuenca es el área geográfica donde el agua se mueve a través de una red de drenaje o vías, ya sean subterráneas o en la superficie (Horner et al., 1997). La cuenca es la unidad apropiada para realizar planificación y gestión participativa, debido a que los procesos que se desarrollan en las partes altas de la cuenca tienen efectos en la partes bajas, ya que el flujo de agua se da en una sola dirección, además dentro de la cuenca se dan una serie de procesos ecológicos y sociales que se pueden analizar de manera integral (Dourojeanni y Jauravlev, 2001: 7). En países europeos, como España, se han implementado modelos de gestión integrales de cuencas hídricas (Directiva Marco de Agua, 2000), en lo que se incluye la participación ciudadana para diseñar políticas y acuerdos que promuevan el manejo sustentable del agua, como el caso de La nueva cultura del agua (Barkin, 2007: 3).

En América Latina la gobernanza del agua se observa en varios procesos participativos locales, como el proyecto Plan en Bolivia, que promueve la participación local en una cuenca para elevar la calidad de vida de los usuarios (Vacaflores, 2000). Otro ejemplo es la experiencia de la Iniciativa de Programa Minga del Centro Internacional de Desarrollo de Investigaciones en Chile, que propone el empoderamiento ciudadano como una estrategia participativa para crear oportunidades para todos los

${ }^{2}$ El concepto de gobernanza efectiva del agua surge como un diálogo propuesto en el Tercer Foro Mundial del Agua en Kioto, Japón, en 2003, por instituciones como GWP, UNDP e ICLEI (International Council for Local Environmental Initiatives) como una estrategia para alcanzar el manejo sustentable del agua (GWP, 2003).

${ }^{3}$ La nueva cultura del agua (NCA) es un planteamiento surgido como rechazo popular a los planes de trasvases del río Ebro, en España. Este movimiento logró colocar los problemas de gestión del agua en la agenda nacional promovida por Pedro Arrojo, galardonado con el Premio Golman del Medio Ambiente en 2003, y se basa en principios básicos como: el agua como derecho humano, el agua para las necesidades ambientales, el agua para usos sociales y comunitarios y el agua para el desarrollo económico (Barkin, 2007). 
usuarios (IDRC, 2003). En Cochabamba, Bolivia, la crisis del agua está promoviendo algunos procesos de gobernanza y movilizaciones sociales que permiten cambiar el sistema de administración del agua (Hoffmann et al., 2006).

En los últimos años, México ha tenido procesos de restructuración de la gestión del agua, se han transformado los marcos legal e institucional para realizar procesos de descentralización. La Ley de Aguas Nacionales (LAN), publicada en 1992 y reformada el 29 abril de 2004, ha avanzado al estipular la unidad de gestión del agua a partir de cuencas hidrológicas, y en consecuencia en la administración del agua desde la perspectiva de cuenca. Por esto se crearon los organismos de cuencas y los consejos de cuencas (LAN, cap. III bis). Estos consejos son instancias de coordinación y concertación entre la Comisión Nacional del Agua (Conagua), las dependencias y entidades de las instancias federal, estatal y municipal, así como con los representantes de los usuarios de la cuenca (Art. 13, LAN). Para su funcionamiento, los consejos de cuenca pueden contar con organizaciones auxiliares a nivel de subcuenca, microcuenca y/o acuífero, denominadas respectivamente, comisiones de cuenca, comités de cuenca y comités técnicos de aguas subterráneas (Cotas).

En México, los proyectos de descentralización del agua promovieron la regulación de los distritos de riego por los usuarios y los sistemas de agua potable administrados por los estados y municipios. Aunque existen estos procesos de descentralización, los consejos han promovido la participación ciudadana; sin embargo, la toma de decisiones para la gestión integrada de los recursos hídricos se sigue concentrando en las instancias de gobierno (Cotler, 2004; Castro et al., 2004; Wilder y Romero, 2006).

\subsection{El marco analítico de la gobernanza (MAG)}

Un enfoque alternativo para analizar la nueva gobernanza lo propone Hufty (2004) mediante el marco analítico de la gobernanza (MAG), desarrollado recientemente en el programa NCCR North-South en el Graduate Institute of International and Development Studies (IHEID) de la Universidad de Ginebra, Suiza. El MAg se diseñó como herramienta práctica para analizar y comprender los contextos sociopolíticos de las investigaciones en este centro de investigación. Un rasgo central de la metodología son los diferentes elementos analíticos o dimensiones observables de la gobernanza: el problema, los actores, las normas sociales, los puntos nodales y los procesos. La configuración específica de estos elementos producen distintos tipos de gobernanza y, por ende, de ciudadanía, por tanto, el futuro del MAG se relaciona con sus ensayos empíricos (Hufty et al., 2006). 
Para Hufty la primera categoría analítica en los procesos de gobernanza es la identificación del problema. Un problema nace en el momento de una confrontación o conflicto que debe ser atendido y que concierne a asuntos públicos. Otra categoría analítica es el reconocimiento de actores sociales (stakeholders) involucrados en el problema. La definición de actor es "todo individuo, organización o grupo con recursos de poder suficientes para impedir o perturbar el funcionamiento de las reglas o procedimientos de toma de decisiones y de soluciones de conflictos colectivos" (Prats, 2003: 243). Hufty retoma este concepto y clasifica a los actores en tres grupos de acuerdo con su nivel de influencia y poder: los actores estratégicos (tienen poder de decisión y generan movilizaciones sociales), los actores relevantes (tienen poder de decisión y dinero y se ven involucrados en algunas instituciones) y los actores secundarios (no tienen poder para cambiar las reglas ni los procesos establecidos).

Los puntos nodales o interfaces sociales se entienden como los sitios de conflicto, incompatibilidad y negociación. Estos espacios pueden ser físicos o virtuales, formales e informales y en ellos los actores exponen sus discursos y son escuchados, proponen acuerdos y estrategias de acción (Long, 2003). Otra categoría, además de los puntos nodales, son los procesos (estados) por los cuales pasa un sistema, es decir, su historia y evolución de un conflicto o problema hacia factores favorables de cambio. El análisis de los procesos de cambio busca identificar los patrones de evolución de los puntos nodales, la trama de interacciones entre actores y su relación con los cambios de las reglas del juego. La última categoría analítica se refiere a las normas, es decir, la construcción de los arreglos y acuerdos entre actores. Las normas asumen un doble rol en el análisis de la gobernanza, orientan el comportamiento de los actores y son modificadas por la acción colectiva. Las normas se pueden identificar en distintos niveles (multiniveles), desde donde se originan hasta donde se aplican (en los ámbitos internacional, nacional, estatal y local) (Hufty et al., 2006).

\section{Diseño del estudio}

El análisis de la gobernanza se realizó asumiendo la postura teórica de Hufty (2004), el MAG, la cual consiste en identificar los problemas, los puntos nodales, los actores, las normas y los procesos. Estos procesos de gobernanza se analizaron en torno al manejo del agua que realizaron los usuarios en las comunidades de microcuenca El Cangrejo, durante el año 2008. La investigación se realizó en cuatro etapas:

1) El análisis de los problemas del agua en la cuenca consistió en describir la situación socioambiental en relación con el manejo del 
agua. Se consultó información secundaria y cartografía de la cuenca en bases de datos (INEGI, 2005).

2) Para identificar a los actores y sus procesos fue necesario realizar entrevistas semiestructuradas con preguntas abiertas sobre los problemas del agua, su participación en la solución de los conflictos, así como los principales acuerdos tomados para su uso. Las entrevistas se realizaron a las siguientes autoridades municipales: regidor de ecología, administrador de agua potable y alcantarillado, director de ecología, director de fomento agropecuario y delegados municipales.

3) Puntos nodales y sus normas. Las entrevistas realizadas a los actores ayudaron a conocer los espacios donde discuten el tema del agua. Analizamos las normas mediante consulta de leyes federales y estatales, reglamentos municipales y acuerdos tomados en las juntas de cabildo, reuniones ejidales y en el consejo municipal de desarrollo rural en el tema del agua. Éstos se obtuvieron por solicitud directa en las oficinas del ayuntamiento.

4) Percepciones de los usuarios en relación con el agua y la participación social. Este apartado contribuye a explicar los procesos de gobernanza percibidos desde las comunidades involucradas. Por tanto, se aplicaron encuestas en las comunidades con mayores problemas o conflictos por el agua. Las localidades elegidas para aplicar las encuestas fueron Ayutita, El Jacalote y La Lima. Se encuestó 10\% ( $\mathrm{n}=20)$ del total de viviendas particulares habitadas. $\mathrm{El}$ instrumento de medición fue una encuesta compuesta por 15 preguntas, la variable dependiente fue la participación social y las variables independientes la edad, sexo, localidad, actividad económica, disposición de agua, calidad del agua y pago de servicio.

\section{Descripción del área de estudio de la microcuenca El Cangrejo}

La microcuenca El Cangrejo se localiza en el occidente de México, en el estado de Jalisco, municipio de Autlán de Navarro, en las coordenadas geográficas $19^{\circ} 45^{\prime} 48.48^{\prime \prime}$ a $19^{\circ} 52^{\prime} 4.78^{\prime \prime}$ de latitud norte y $104^{\circ} 28^{\prime}$ $8.00^{\prime \prime}$ a $104^{\circ} 19^{\prime} 1.14^{\prime \prime}$ de longitud oeste. Esta microcuenca pertenece a la región hidrológica de la cuenca del río Ayuquila-Armería, en la costa sur de Jalisco, su temperatura media es de $23^{\circ} \mathrm{C}$, la precipitación promedio anual de $1,082 \mathrm{~mm}$, a una altitud de $1,831 \mathrm{msnm}$, tiene una área de $91.24 \mathrm{~km}^{2}$ y una longitud de $17.51 \mathrm{~km}$, y pendientes con una inclinación de $8.67 \%$. El canal principal de la microcuenca es el arroyo El Cangrejo, con una longitud de $18.1 \mathrm{~km}$, se considera una cuenca pequeña, tipo oval 
redonda a oval-oblonga, con corrientes efímeras, intermitentes y perennes (Meza, 2008).

Los principales problemas ambientales de la microcuenca El Cangrejo son: la erosión ligera y alta en $70 \%$ del territorio, la pérdida de cobertura vegetal por actividades antropogénicas como el sobrepastoreo y la tala de árboles en las partes altas de la cuenca (zonas de recarga hídrica y cabecera de cuenca). Las consecuencias de estos problemas son inundaciones en las localidades de las partes bajas de la cuenca. Otro problema que se identificó es la contaminación del agua del arroyo, que aunque no es grave se puede incrementar, principalmente por las descargas de residuos de los establos ganaderos ubicados en los márgenes del arroyo El Cangrejo.

La población de la microcuenca se divide en dos: una zona urbana compuesta por 15 colonias de la cabecera municipal, con una población total de 40,791 habitantes, y la zona rural localizada en parte alta de la microcuenca la conforman las localidades de El Jalocote, La Lima de los Gómez, Ayutita y el Volantín, con una población total de 723 habitantes (INEGI, 2005). Las localidades de El Jalocote y La Lima presentan altos niveles de marginación. Las actividades económicas de la población rural se basan en la agricultura (cultivos de hortalizas, caña y maíz) y la ganadería (granjas de ovinos, bovinos, porcinos y aves). La población urbana se dedica principalmente al comercio. La tenencia de la tierra se divide en 16,682 ha de propiedad privada, 42,632 ha de propiedad ejidal y 37,926 ha de propiedad comunal (INEGi, 2005). En el mapa i se muestra la localización de la microcuenca El Cangrejo.

\section{Conflictos por el uso del agua en las comunidades rurales de la microcuenca El Cangrejo}

Los pobladores rurales de la microcuenca El Cangrejo, tienen graves problemas de abastecimiento de agua potable y falta de agua para uso agrícola y pecuario, debido a que el gobierno municipal destina parte del acuífero a los usos públicos que se derivan del arroyo El Cangrejo. El agua de las corriente superficiales de la cuenca se destina principalmente al uso público urbano, con un volumen aproximado de $892,504 \mathrm{~m}^{3}$, que representa $15 \%$ del total municipal. La explotación del agua subterránea pertenece al acuífero de Autlán, que comprende un total de 260 pozos, de los cuales 141 corresponden a uso agrícola. Los pozos tienen una condición de subexplotación (CNA, 2001). En el mapa II se observa la microcuenca El Cangrejo y cómo la explotación de agua superficial se realiza en las partes altas de la cuenca, en las localidades de La Lima y El Jalocote. En el mapa III se muestran los pozos de abastecimiento de agua ubicados, en su mayoría, en la parte baja de la cuenca, principalmente en el municipio de Autlán (CNA, 2001). 


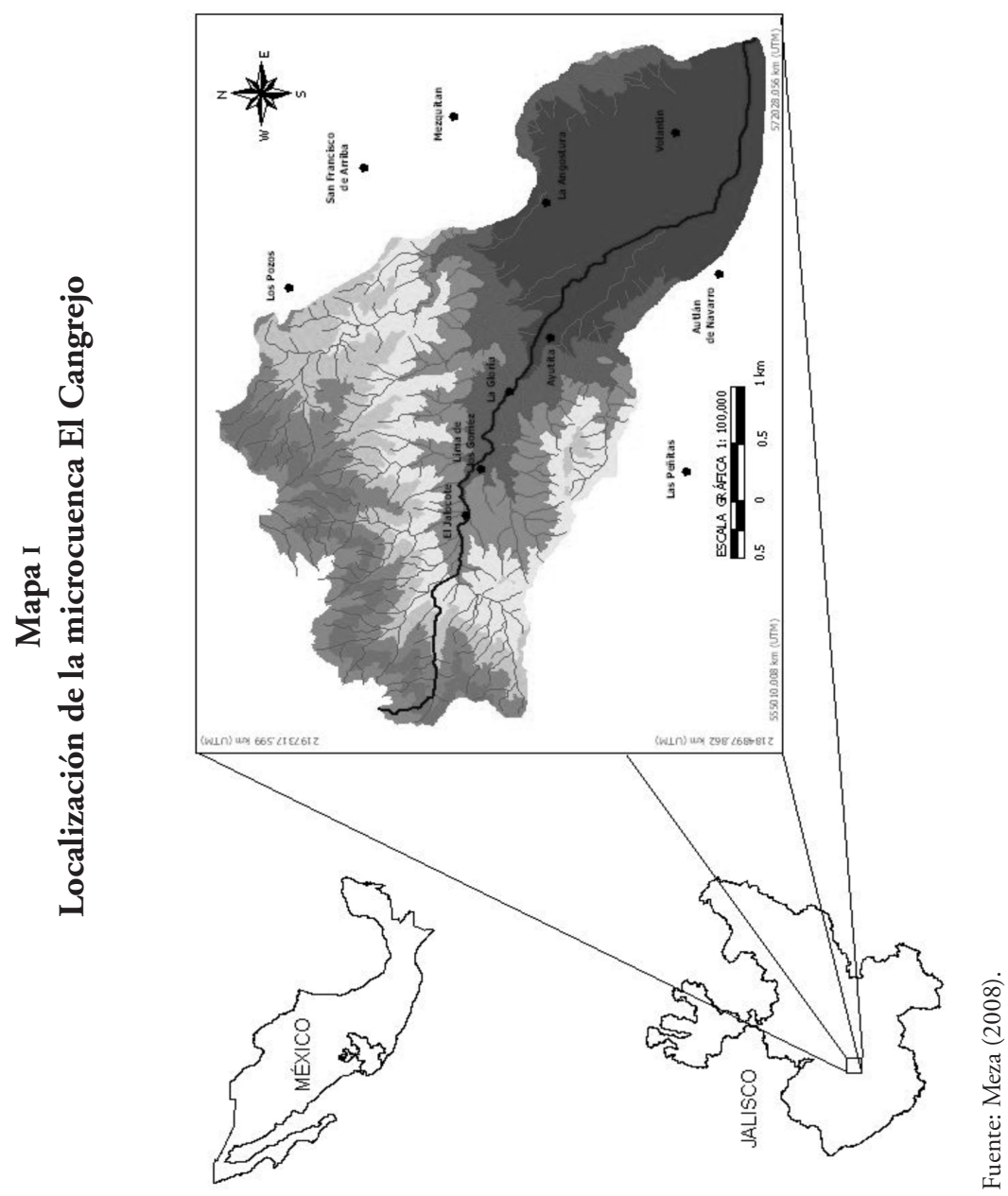




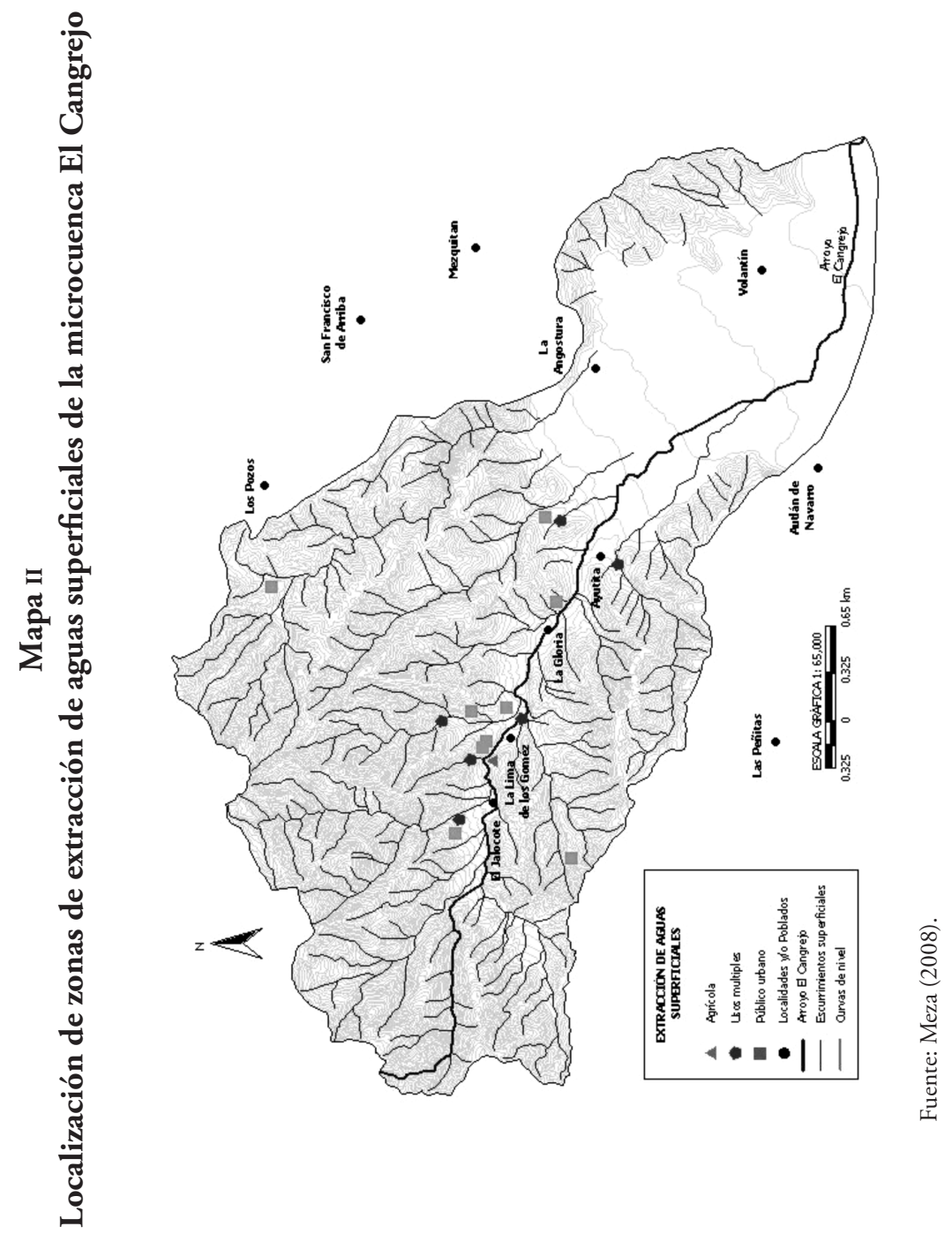




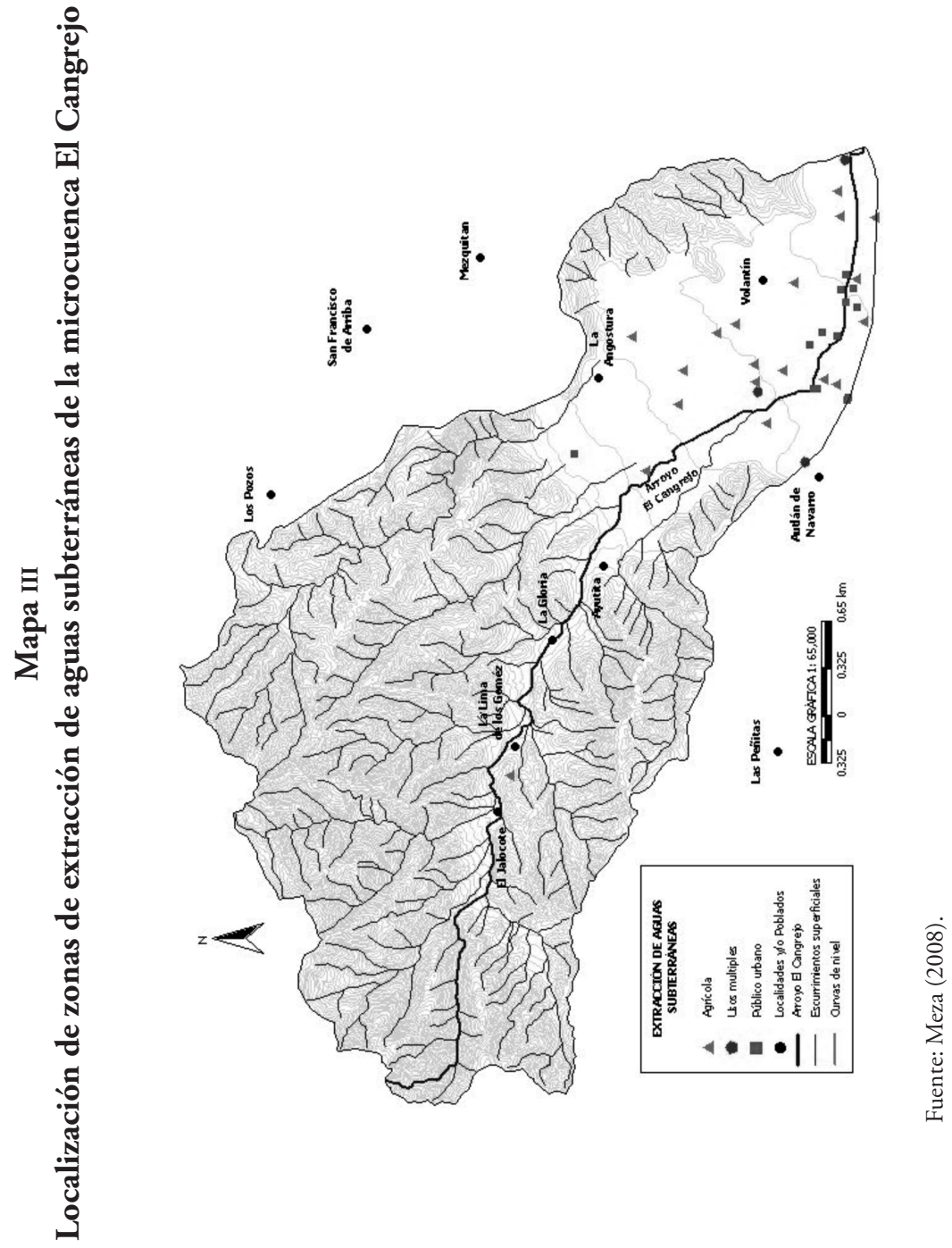


El conflicto por acceso al agua se observó en las localidades de El Jalocote y La Lima, que por ser zonas marginadas carecen de servicios básicos de agua potable y alcantarillado (INEGI, 2005). La demanda de agua para satisfacer sus necesidades domésticas y agropecuarias es alta, por esto elaboraron sus propios sistemas de conducción de agua, la cual toman del arroyo El Cangrejo. Sin embargo, el acceso es limitado porque sólo disponen de agua entre dos y tres horas al día. Es importante mencionar que también existen canales de riego en los cultivos de caña y hortalizas sin uso limitado de agua. Además, como no cuentan con sistemas de alcantarillado, vierten su residuos directamente al arroyo.

El conflicto se incrementa porque el gobierno municipal de Autlán también toma agua directamente del arroyo El Cangrejo, para abastecer las viviendas de 15 colonias de la parte baja de la cuenca. Esta situación ha suscitado la inconformidad de los pobladores de las partes altas de la microcuenca, ya que la sobreexplotación que hace el gobierno municipal del agua superficial del arroyo provoca que éste se seque y las poblaciones rurales de El Jalocote y La Lima no tengan suficientes recursos para satisfacer sus actividades.

\section{Los actores involucrados}

Los actores identificados en la microcuenca se pueden clasificar en estratégicos, relevantes y secundarios, de acuerdo con la metodología MAG.

Los actores estratégicos. En las comunidades rurales de El Jalocote y Ayutita es evidente la acción de los ejidos como grupos organizados de mayor importancia en la microcuenca. Los ejidatarios tienen el control y administración del agua, su organización es autogestiva, actúan con recursos propios, no interviene el gobierno en la toma de decisión y cuentan con suficiente poder para promover movilizaciones sociales. Recientemente solicitaron al gobierno municipal la construcción de una presa, Las Mancornadas, para solucionar sus conflictos de acceso al agua, sin embargo, aún no reciben una respuesta.

Los actores relevantes. En la microcuenca, aunque los usuarios se organizan de manera independiente, en cualquier momento el Estado puede intervenir a partir del marco jurídico, aunque hasta ahora no lo ha hecho a pesar de que cuenta con los recursos y el poder suficiente. Las instituciones formales como la CNA no intervienen en ningún sentido en este conflicto. La Secretaría de Desarrollo Rural (Seder) y el programa de microcuencas Fideicomiso de Riesgo Compartido (Firco) participan asignando un técnico para promover proyectos productivos en zonas marginadas, pero no interviene en la organización del agua de las comunidades. 
La Comisión Estatal del Agua (CEA) sólo se encarga de atender la solicitud de los ejidatarios respecto a la construcción de la presa Las Mancornadas. El gobierno municipal actúa en el territorio de la microcuenca asignando el agua para abastecer a la población urbana, pero no interviene en los acuerdos del comité de agua del ejido. Otro actor clave es la Universidad de Guadalajara, por medio del Centro Universitario de la Costa Sur (Cucsur), en Autlán de Navarro, que aunque cuenta con recursos para investigación y técnicos especializados en hidrología, tampoco interviene en el conflicto. La Junta Intermunicipal de la Cuenca del Río Ayuquila (JIRA) es un organismo público descentralizado conformado por gobiernos municipales, estatales y federales, asesora a diferentes municipios de la región para la gestión del agua, pero en el ámbito local no incide en la microcuenca. Por último, el actor formal representado por el Consejo de Cuenca del Río Ayuquila-Armería no interviene de ninguna forma en este problema.

Los actores secundarios son aquéllos que no tienen ningún poder para cambiar las reglas y procesos establecidos. En este caso podemos mencionar principalmente a los usuarios pobladores no organizados.

\section{Los puntos nodales}

En nuestro estudio identificamos tres espacios físicos donde se analizan los temas referentes al manejo del agua con intereses particulares, sin tener un espacio de discusión común.

Comité de Agua de la localidad de La Lima. Es una organización local formada por ejidatarios y usuarios, y se encarga de administrar el agua para usos agrícola y doméstico que proviene de la parte superficial del arroyo El Cangrejo. El comité establece acuerdos sobre los horarios de uso de agua (dos o tres horas por día). En este espacio no interviene ninguna otra institución. Se consideran dueños del agua y no están dispuestos a compartirla con otras comunidades.

Reuniones ejidales en Ayutita y El Jalocote. En ellas sólo participan propietarios de ejidos, con fines de distribución agrícola, muchos de ellos crean su propia infraestructura para llevar agua a sus hogares y a sus parcelas, tomando agua directamente del arroyo o de pozos. Debido al problema de falta de abastecimiento de agua, han promovido ante las autoridades municipales y estatales que se construya la presa Las Mancornadas (no existe un comité de aguas superficiales, Cotas).

Reuniones municipales de cabildo. Para cumplir las funciones del gobierno municipal, cada mes se realizan reuniones de cabildo para tratar diferentes problemáticas. A partir de 2007, el tema del agua tiene un trato especial por parte de los regidores y el presidente municipal, debido 
al incremento en la demanda de agua y la falta del recurso. Sin embargo, aún no se tiene un plan de manejo integral de la microcuenca que garantice el acceso al agua en el futuro.

\section{El marco institucional y normativo de la cuenca}

Los problemas identificados en la cuenca El Cangrejo se encuentran en una serie de normas y acuerdos e instituciones creadas para regular el uso y la distribución del agua.

Ámbito nacional. La Ley de Aguas Nacionales (LAN, 2004), en el artículo 13, menciona que los consejos de cuenca se formaron como espacios de integración mixta. Según la ley, estos consejos se crearon para promover y facilitar la participación de la sociedad en la planeación, toma de decisiones, ejecución, evaluación y vigilancia de la política nacional hídrica (LAN, 1992, Art. 14 bis). El Consejo de Cuenca Ayuquila-Armería no está articulado con la microcuenca El Cangrejo, debido a que no existen mecanismos para integrar a este nivel la participación de los usuarios en la toma de decisiones.

La LAN también establece la formación de distritos de riego mediante la conformación de asociaciones de usuarios; en este caso existen distritos de riego pero generalmente los problemas del agua se tratan en los ejidos, que están regulados por la Ley Agraria. La Ley General del Equilibrio Ecológico y Protección al Ambiente (LGEEPA), publicada en 1988, promueve el aprovechamiento sustentable del agua y los ecosistemas acuáticos (título tercero, capítulo I), por tanto, la LAN se sujeta a esta ley en varios de sus artículos. El tema de la calidad del agua para abastecimiento y almacenamiento para uso y consumo, se trata en la Ley General de Salud (Capítulo Iv, artículos 118, 119, 121,122). En el caso de la microcuenca El Cangrejo, esta normatividad en materia ambiental y de salud no la aplican ni regulan las instituciones responsables.

Ámbito estatal. La CEA, por medio de la Ley del Agua para el Estado de Jalisco y sus Municipios, coordina y planifica los usos del agua en la entidad con funciones de autoridad administrativa, en muchos casos se vinculan con los municipios para construir presas y plantas de tratamiento. Asimismo se coordina con la Seder para promover el desarrollo de infraestructura hídrica rural. En materia de administración de agua para uso público urbano, la Ley de Servicios de Agua Potable y Alcantarillado en el Estado de Jalisco permite la correcta administración, distribución y saneamiento de las aguas de uso público urbano para sus municipios.

Ámbito municipal. El marco jurídico del gobierno y la administración municipal se articulan con las leyes nacionales y estatales, mediante la formulación de reglamentos locales que facilitan el cumplimiento de 
estas leyes. En el municipio de Autlán de Navarro, la función de la Dirección de Agua Potable y Alcantarillado es dar servicios de agua potable, drenaje, alcantarillado, tratamiento y disposición de aguas residuales. Para ello se basan en el Reglamento de Agua Potable y Alcantarillado y en el Reglamento de Medio Ambiente y Ecología. Los cobros del servicio de agua a los pobladores se regulan por medio de la Ley de Hacienda $\mathrm{Mu}-$ nicipal y la Ley del Gobierno y la Administración Pública. En este contexto jurídico se percibe que aunque existen los reglamentos para regular y vigilar el manejo del agua, no se aplican sobre todo en lo referente a desperdicio de agua de la población rural y urbana, en la promoción de cultura del agua, además no se tiene personal capacitado en materia de educación ambiental para el cuidado del agua. Tampoco existe una visión integral del manejo de la cuenca.

Acuerdos sociales informales. Los pobladores de las zonas rurales, principalmente en El Jalocote y La Lima, se organizan de manera independiente, es decir, no interviene ningún nivel de gobierno. El comité del agua tiene un reglamento interno que establece los horarios de suministro de agua a la comunidad, así como los cobros para el mantenimiento de redes de distribución. Aunque el suministro es insuficiente, la comunidad asume el costo que varía entre 50 y 100 pesos anuales.

\section{Percepciones de los usuarios en la microcuenca}

Las encuestas realizadas en las comunidades rurales de El Jalocote (aguas arriba de la cuenca), La Lima (parte media) y Ayutita (parte baja) muestran aspectos relevantes. Los encuestados tienen una edad promedio de 40-50 años; $70 \%$ son mujeres amas de casa; la escolaridad promedio es primaria, aunque la población de Ayutita tiene escolaridad promedio de secundaria, tal vez por su cercanía con zona urbana. Respecto a la percepción de los problemas, alrededor de $80 \%$ de la población de La Lima y El Jalocote consideran que la calidad del agua es buena, incluso la utilizan para beber. Sin embargo, en Ayutita $60 \%$ opina que la calidad es regular, por tanto, consumen agua de garrafón.

El principal problema percibido por $80 \%$ de la población es la falta de agua en temporadas de estiaje (secas), de abril a junio, cuando el arroyo El Cangrejo se seca. En El Jalocote y La Lima la población opina que el servicio de suministro de agua es adecuado, aunque sea limitado. Los comités de agua de las localidades realizan un cobro anual por el servicio de agua (tuberías, insumos), la tarifa más alta es de 120 pesos en El Jalocote, seguido de 100 pesos para Ayutita y 50 en La Lima. Al respecto, $90 \%$ de los entrevistados consideran justo o barato el precio. 
En las tres localidades consideran que el gobierno municipal es bueno (80-90\%), aunque no conocen y no participan en los programas propuestos por las autoridades. Las respuestas sobre el nivel de participación social fueron diferentes entre localidades, los habitantes de La Lima y Ayutita muestran apatía por participar en los consejos y comités de agua. Del total de entrevistados, a menos de la mitad le interesa participar, cabe mencionar que quienes contestaron de esta manera fueron $80 \%$ mujeres y jóvenes. En cambio, en El Jalocote 100\% de los entrevistados están interesados o participan activamente en los comités de agua. En la gráfica I se muestran los niveles de participación social por localidad.

\section{Gráfica I}

Participación social en las comunidades de la microcuenca El Cangrejo

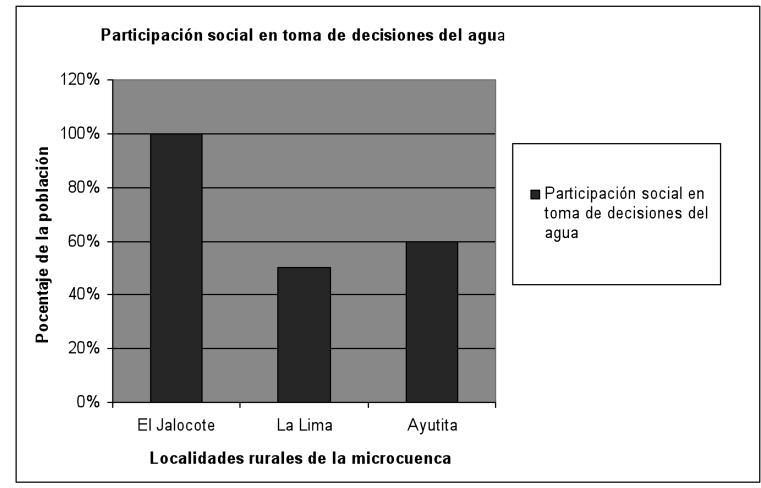

Fuente: Meza (2008).

\section{Discusiones y conclusiones}

El presente trabajo permitió identificar los procesos de gobernanza en la gestión del agua en la microcuenca El Cangrejo, mediante la identificación de los problemas a partir de una descripción física y social del área de estudio. La metodología MAG propuesta por Hufty, nos permitió analizar los componentes de la gobernanza: la identificación de problemas, los actores, las normas y los procesos que se desarrollan en la toma de decisiones. Consideramos que es una herramienta útil para analizar temas ambientales, concretamente el manejo y la gestión del agua.

De acuerdo con Kooiman (2003), la gobernanza es el conjunto de interacciones entre actores públicos y privados orientadas a resolver sus problemas sociales, para crear oportunidades en un marco normativo. En 
el análisis que se presentó en este artículo, los procesos de gobernanza son incompletos y con fallas, porque no involucran a todos los actores y no se consideran la normas establecidas formalmente en la toma de decisiones.

Con respecto al problema de falta de agua en estas poblaciones, existe participación social para tomar acuerdos, pero no participa ninguna instancia gubernamental, por tanto, las leyes establecidas en estas localidades no se aplican. En cuanto al segundo problema, la demanda de agua para actividades agropecuarias, al igual que en el anterior, aunque tienen reuniones ejidales normadas formalmente, no son espacios donde se promueva la participación de actores de gobierno, ni se cuenta con técnicos especializados en agua, sólo se persiguen intereses privados. En el caso de la demanda de agua para las zonas urbanas sucede todo lo contrario, se toman decisiones en la sala de cabildo del ayuntamiento de acuerdo con el marco jurídico e institucional, pero no existe participación ciudadana, ni siquiera de los ejidatarios como en la toma de decisiones. Finalmente, el problema relacionado con el deterioro físico de la cuenca no tiene un punto nodal, aunque sí hay un marco normativo para prevenir el deterioro ambiental de ésta (LAN, LGEEPA) no se tiene un consejo a nivel de microcuenca que tenga una visión integral del manejo de los recursos hídricos.

Actualmente diversas organizaciones internacionales proponen la gestión integral de los recursos hídricos (GIRH), que establece precisamente que el agua se debe manejar en cuenca hídrica, ya que esto permite un mejor análisis de los problemas que existen en una región determinada (PNUMA, 2000). El análisis de la gobernanza del agua mediante el MAG contribuye a desarrollar programas para la gestión integral de los recursos hídricos, debido a que se analizan los actores clave en la gestión del agua, además se describen las capacidades institucionales de los tres niveles de gobierno para atender los problemas y desarrollar planes y acciones integradas.

En la microcuenca El Cangrejo hay una estructura normativa e institucional apta para desarrollar planes integrales de manejo del agua, pero no se cuenta con capacidades técnicas suficientes que atiendan la promoción de programas de educación ambiental, cultura del agua, protección de los suministros, control de la erosión y las inundaciones, así como fomento de valores estéticos del agua. La falta de coordinación entre los diferentes niveles de gobierno, la ausencia de integración entre instituciones y la participación ciudadana de actores locales rurales tienen como consecuencia que no exista un manejo adecuado de los recursos hídricos.

Por tanto, es importante fomentar las organizaciones rurales, crear fortalezas locales internas, así como concientizar sobre la problemática ambiental, esto permitirá que los grupos rurales generen acciones y políticas de desarrollo y conservación. Es importante que los actores relevan- 
tes, es decir, las instituciones de gobierno, intervengan en la toma de decisiones del comité del agua de las localidades rurales en El Jalocote y en La Lima de los Gómez. Consideramos que aunque los ejidatarios y los pobladores pueden resolver sus problemas de manera autónoma, es importante y fundamental la intervención y la vigilancia de las instituciones de gobierno para proteger los recursos naturales que son de interés público y no privado.

En general los resultados de este trabajo demuestran que existen elementos positivos para promover procesos efectivos de gobernanza en la gestión integral de la microcuenca. Sin embargo, se deben considerar algunos problemas y fallas que identificamos. Por ejemplo, el problema de falta de agua en las poblaciones rurales, ya que existe participación social para tomar acuerdos, pero no involucran ninguna instancia gubernamental, por tanto las leyes establecidas en estas localidades no se aplican.

En el caso de la demanda de agua para actividades agropecuarias, al igual que en el anterior, aunque tienen reuniones ejidales (institución formal), no son espacios donde se promueva la participación de actores de gobierno ni de técnicos especializados en agua, sólo se persiguen intereses privados. En el caso de la solución de problemas de demanda de agua para zonas urbanas, esto se lleva a cabo en la sala de cabildo del ayuntamiento de Autlán de Navarro, siguiendo el marco jurídico e institucional formal. Sin embargo, aunque existen mecanismos para la consulta de problemas públicos, no se logran articular con los comités de agua en los ejidos de la cuenca El Cangrejo aguas arriba.

Acerca de los problemas relacionados con el deterioro físico y la sobreexplotación de agua, no tiene un punto nodal o espacio (físico o virtual) en el que los diferentes actores expongan sus problemas y se propongan planes de manejo integral de la microcuenca. Aunque existe un marco normativo e institucional (Consejo de Cuenca Ayuquila-Armería, LAN, LGEEPA, Seder-Firco/Microcuencas, JIRA, Universidad de Guadalajara) que puede incidir y apoyar, no se identificó ningún planteamiento de coordinación interinstitucional. Ninguno de los tres puntos nodales identificados (comité de agua, reuniones ejidales y sesiones de cabildo en ayuntamiento) se interrelacionan, cada uno satisface sus necesidades sin tener una visión de cuenca y planeación integral, por lo que está en riesgo el acceso al agua para el futuro.

Se puede considerar que en la microcuenca El Cangrejo hay elementos de gobernanza del agua, como la estructura normativa e institucional desarrollada en los ámbitos estatal y federal (consejos de cuencas, Cotas, etc.), pero no existen estos comités constituidos a nivel local de microcuenca. Por tanto, es importante implementar estos instrumentos de participación social en la región, aprovechando las oportunidades y for- 
talezas del gobierno municipal actual de Autlán de Navarro, que muestra apertura y aceptación de la mayoría de los usuarios rurales de la microcuenca, como se demostró en nuestra encuesta en las comunidades.

Por tanto, es importante que el gobierno intervenga y participe en la gestión del agua fomentado en las organizaciones rurales fortalezas locales internas, así como crear conciencia sobre la problemática ambiental, esto permitirá que los grupos rurales generen acciones y políticas de desarrollo y conservación del agua en la microcuenca. Es importante que los actores relevantes, es decir, las instituciones de gobierno, intervengan en la toma de decisiones que se están desarrollando en el comité del agua de las localidades de El Jalocote y en La Lima de los Gómez. Consideramos que aunque los ejidatario y los pobladores pueden resolver sus problemas de manera autónoma, es importante y fundamental la intervención y la vigilancia de las instituciones de gobierno para proteger los recursos naturales, ya que éstos son de carácter público, no privado.

\section{Agradecimientos}

Queremos agradecer a la Universidad de Guadalajara, sobre todo al Departamento de Ecología y Recursos Naturales del Centro Universitario de la Costa Sur, por su apoyo institucional para realizar el trabajo; y en particular, a Aída Alejandra Guerrero-de León, por permitir llevar a cabo esta investigación como parte de un tema de la tesis doctoral del programa Bemarena. Asimismo agradecemos al programa Swiss National Centre of Competence in Research (NCCR North South), por medio del WP1/ JACS-CCA, por su apoyo económico e institucional.

\section{Bibliografía}

Aguilar Villanueva, Luis Felipe (2006), Gobernanza y la gestión pública, Fondo de Cultura Económica, México.

Barkin, David (2007), La gestión del agua urbana en México. Retos, debates y bienestar, Universidad de Guadalajara, México.

Castro, José Esteban, Karina Kloster y María Luisa Torregrosa (2004), "Ciudadanía y gobernabilidad en México: el caso de la conflictividad y la participación social en torno a la gestión del agua”, en Blanca Jiménez y Javier Alcocer (coords.), El agua en México visto desde la academia, Academia Mexicana de Ciencias, México, pp. 201-232. 
CNA (Comisión Nacional del Agua) (2001), Programa Hidráulico Nacional. El agua: un recurso estratégico y de seguridad nacional 20012006, CNA, México.

Cna (Comisión Nacional del Agua) (2008), Estadísticas del agua en México 2008, Semarnat, México.

Córdova Bojórquez, Gustavo, María de Lourdes Romo Aguilar y Sergio Peña Medina (2006), "Participación ciudadana y gestión del agua en el valle de Juárez, Chihuahua”, Región y Sociedad, xviII (35), El Colegio de Sonora, México, pp. 75-105.

Cotler, Helena (comp.) (2004), El manejo integral de cuencas en México: estudios y reflexiones para orientar la politica ambiental, SemarnatINE, México.

Delgado, Luisa E., Pamela L. Bachmann y Bárbara Ońate (2007), “Gobernanza ambiental: una estrategia orientada al desarrollo sustentable local a través de la participación ciudadana", Ambiente y Desarrollo, 23 (3), pp. 68-73.

Directiva Marco de Agua 2000/60/CE del Parlamento Europeo y del Consejo (2000), de 23 de octubre, por la que se establece un marco comunitario de actuación en el ámbito de la política de aguas Dol 327 de 22/12/2000.

Dourojeanni, Axel y Andrei Jouravlev (2001), Crisis de gobernabilidad en la gestión del agua. Desafíos que enfrenta la implementación de las recomendaciones contenidas en el capitulo 18 del Programa 21, Cepal, Santiago de Chile.

Galvin, Marc y Haller Tobias (2008), "People, Protected Areas and Global Change: Participatory Conservation in Latin America, Africa, Asia and Europe. Perspectives of the Swiss National Centre of Competence in Research (NCCR) North-South", Geographica Bernensia, 3, Universidad de Berna, Berna, pp. 145-153.

Gerritsen, Peter, Alma Lomelí Jiménez y Claudia Ortíz Arrona (2005), "Urbanización y problemática socioambiental en la costa sur de Jalisco, México. Una aproximación”, Sociedad y Territorio, 17 (33), El Colegio de Sonora, México, pp. 107-137. 
GWP (Global Water Partnership) (2003), "Dialogue on Effective Water Governance: Action through Partnership (in Central and Eastern Europe)", $3^{\text {rd }}$ World Water Forum, Kyoto, Japón, 16-23 de marzo.

GWP (Global Water Partnership) (2009), "Integrated Water Resources Management in Practice: Better Water Management for Development por Roberto Lenton and Mike Muller of the Global Water Partnership Technical Committee and published by Earthscan GwP, Estocolmo, World Water Week, http://www.gwpforum.org/ servlet/PSP?iNodeID=215\&itemId=889, 5 de agosto de 2008 .

Hoffmann, Sabine, Bernardo Rozo, Luis Tapia Mellea y Jorge Viana (2006), La reconstrucción de lo público. Movimiento social, ciudadania y gestión de agua en Cochabamba, Muela del Diablo, La Paz, Bolivia.

Horner, Richard Ray, Derek Blake Booth y Amanda Azous (1997), "Watershed determinants in ecosystem functioning", en L. R. Roesner (ed.), Effects of watershed development and management on aquatic ecosystems. American Society of Civil Engineers, Nueva York, pp. 251-274.

Hufty, Mark (2004), Marco conceptual de la gobernanza. Una propuesta para concretizar el concepto de la gobernanza, Instituto Universitario de Estudios del Desarrollo, Ginebra.

Hufty, Mark, Eduardo Bascolo y Roberto Bazzani (2006), "Gobernanza en salud: un aporte conceptual y analítico para la investigación", Cad. Saúde Pública, 22, Río de Janeiro, pp. 35-45.

IDRC (International Development Research Centre) (2003), “El agua y la gobernanza. Contribución de la investigación en los Andes", Iniciativa del Programa Minga del Centro Internacional de Investigaciones para el Desarrollo, Otawa.

INEGI (Instituto Nacional de Estadística, Geografía e Informática) (2005), Datos estadísticos del Censo de Población y Vivienda 2005, Municipio de Autlán de Navarro, Jalisco.

Iza, Alejandro Omar y Marta Brunilda Rovere (2006), Gobernanza del agua en América del Sur. Dimensión ambiental, Centro de Derecho Ambiental de la Unión Internacional para la Conservación de la 
Naturaleza y los Recursos Naturales, Gland, Suiza-Cambridge, Reino Unido.

Iza, Alejandro Omar y Grethel Aguilar (2006), Gobernanza de aguas compartidas: aspectos jurídicos e institucionales, Unión Internacional para la Conservación de la Naturaleza y los Recursos Naturales-Mesoamérica, San José, Costa Rica.

Kooiman, Jan (2003), Governing as Governance. Governance, governing and interaction, Sage, Londres.

LAN (Ley de Aguas Nacionales) (2004), Diario Oficial de la Federación, 1 de diciembre de 1992, reformada en 2004.

Long, Norman (2003), "An Actor-Oriented Approach to Development Intervention. In .Rural Life Improvement in Asia”, informe de the Asian Productivity Organization Seminar on Rural Life Improvement for Community Development, Tokio, 22-26 de abril de 2002.

Mayntz, Renate (2001), "El Estado y la sociedad civil en la gobernanza moderna”, ClaD Reforma y Democracia, 21, Caracas pp. 1-8.

Martínez Rivera, Luis Manuel, Eduardo Santana Castellón y Sergio Graf Montero (2002), "Una visión del manejo integrado de cuencas", curso Manejo Integrado de Ecosistemas, Colegio de Postgraduados, Montecillo, México, 25 de febrero-1 de marzo.

Meza Rodríguez, Demetrio (2008), "Elaboración de mapas hidrológicos de la microcuenca El Cangrejo (Programa Arcview)", Universidad de Guadalajara-Centro Universitario de la Costa Sur-Laboratorio de Manejo de Cuenca Hídricas, Autlán de Navarro, Jalisco.

Oliveira Barata, Manuel (2002), Etymologie du terme "gouvernance", Comisión Europea, http://europa.eu.int/comm/governance/docs/ doc5_en.pdf, 2 de febrero de 2008.

Ostrom, Elinor (1990), Governing the Commons. The Evolution of Institutions for Collective Action, Cambridge University Press, Londres.

Prats, Joan Oriol (2003), "El concepto y el análisis de la gobernabilidad", Instituciones y Desarrollo, 14, Institut Internacional de Governabilitat de Catalunya, Barcelona, pp. 239-269, http://www.iigov.org. 
PNUMA (Programa de Naciones Unidas para el Medio Ambiente) (2000), Perspectivas del medio ambiente mundial. Geo-2000, MundiPrensa, México.

Rhodes, Rod (1997), Understanding Governance: Policy Networks, Governance, Reflexivity and Accountability, Open University Press, Buckingham, Reino Unido.

Rogers, Peter (2002), Water Governance in Latin American and the Caribbean, BID, Fortaleza, Brasil.

Simonovic, Slobodan (2000), "Tools for Water Management One View of the Future", Water International, 25 (1), Routledge, pp. 76-88.

Toledo, Alejandro (2002), "El agua en México y en el mundo", Gaceta Ecológica, 64, de INE-Semarnat, México, pp. 9-18.

undP (United Nations Development Programme) (2006), Human Development Report 2006 Beyond Scarcity: Power, poverty and the global water crisis, Bruce Ross-Larson, Meta de Coquereaumont y Christopher Trott (eds.), undp, Nueva York.

unESCo (United Nations Educational, Scientific and Cultural Organization) (2009), World Water Assessment Programme, The United Nations World Water Development Report 3, unEsco-Earthscan, París-Londres.

Vacaflores, Carlos Rivero (2000), "La gestión del territorio: un enfoque a partir de la experiencia del río La Sal, Bolivia. Proyecto Plan Bolivia”, Comunidad de Estudios Jaina, Tarija-Bolivia, ponencia presentada en la conferencia de GLCRSP, México.

Wilder, Margaret y Patricia Romero Lankao (2006), "Paradoxes of Decentralization: Water Reform and Social Implications in Mexico", World Development, 34 (11), Universidad McGill, Quebec, pp. 1977-1995.

wwc (World Water Council) (2000), Report World Water. Vision by William J. Cosgrove and Frank R. Rijsberman, Earthscan Publications Ltd., Londres. 
Recibido: 29 de mayo de 2009 Aceptado: 21 de enero de 2010

Aída Alejandra Guerrero-de León. Es maestra en ciencias por la Universidad de Guadalajara (UG) y licenciada en biología por la misma universidad. Actualmente cursa el doctorado en manejo de recursos naturales en el Instituto Manantlán de Ecología y Conservación de la Biodiversidad (Imecbio) del Centro Universitario de la Costa Sur, Ug. Es becaria del programa suizo National Center of Competence in Research (NCCR) North-South (2007-2009). Sus líneas de investigación se centran en agua y salud. Ha participado en los siguientes foros: "Manejo del agua y sustentabilidad en la cuenca del río Santiago en el Estado de Jalisco", Congreso de la Asociación Mexicana de Ciencias para el Desarrollo Regional, Universidad Autónoma de Aguascalientes, 27-31 de octubre de 2008; "La contaminación del río Santiago y la salud pública en los municipios de El Salto y Juanacatlán”, Congreso Nacional de Medicina Social y Colectiva, Ciudad de México, 24-28 de noviembre de 2008; "Procesos de gobernanza y manejo sustentable del agua en dos cuencas del estado de Jalisco", Taller internacional JACS ACC del programa NCCR North South, 1-5 de octubre de 2007, Tegucigalpa, Honduras.

Peter R. W. Gerritsen. Es doctor en ciencias sociales por la Universidad de Wageningen, Países Bajos, es maestro en ciencias con especialización en agroforestería y forestería social por la misma universidad. Es miembro regular de la Academia Mexicana de Ciencias y pertenece al Sistema Nacional de Investigadores (nivel II). Actualmente es jefe del Laboratorio de Desarrollo Rural, Departamento de Recursos Naturales (Dern), del Instituto Manantlán de Ecología y Conservación para la Biodiversidad (Imecbio), del Centro Universitario de la Costa Sur (Cucsur) de la UG. Sus líneas de investigación son: aspectos sociológicos del manejo de recursos naturales, desarrollo rural endógeno, economía campesina, estrategias campesinas y conservación de la biodiversidad, género y manejo de recursos naturales, globalización, urbanización y respuestas locales, procesos de gobernanza y manejo sustentable de agua, y tenencia de recursos naturales. Sus publicaciones más relevantes son: "Percepciones sobre la degradación ambiental de agricultores orgánicos y convencionales en el ejido La Ciénega, municipio de El Limón, Jalisco, México", Economía, Sociedad y Territorio, viI (25), El Colegio Mexiquense, Toluca, México, pp. 215-239 (2007); "Dinámica espacial y temporal de la ganadería extensiva: estudio de caso de la Sierra de Manantlán en la Costa Sur de Jalisco", Relaciones, Estudios de Historia y Sociedad, 27 (108), pp. 163-189 (2006); "Incorporating principles of sustainable development in research 
and education in western Mexico", Journal of Cleaner Production, 14, Issues 9-11, Guadalajara, pp. 1003-1009 (2006); Dinámica espacial y temporal de la ganadería extensiva: estudio de caso de la Sierra de Manantlán en la costa sur de Jalisco, Universidad de Guadalajara, Jalisco (2008).

Luis Manuel Martínez-Rivera. Es doctor en manejo de cuencas por la Universidad de Utah, cursó la maestría y la licenciatura en ciencias agropecuarias en la Universidad Autónoma de Chihuahua. Actualmente es jefe del Departamento de Recursos Naturales (Dern) del Instituto Manantlán de Ecología y Conservación para la Biodiversidad (Imecbio), de la UG. Su línea de investigación es manejo de cuencas. Sus publicaciones más relevantes son: "Collaborative governance for sustainable water resources management: the experience of the Inter-Municipal Initiative for the Integrated Management of the Ayuquila River Basin, Mexico", Environment and Urbanization, 18 (2) (2006); "Incorporating Principles of Sustainable Development in Research and Education in Western Mexico", Journal of Cleaner Production, 14, pp. 1003-1009 (2006); "Livestock impact on dynamic and structure of tropical dry forest of the Sierra de Manantlan, Mexico", Journal of Food, Agriculture and Environment, 4 (3-4), pp. 266-270. (2006).

Silvia Salcido-Ruíz. Es ingeniero en recursos naturales por la Universidad de Guadalajara y cursa la Maestría en Manejo de Recursos Naturales en el Departamento de Recursos Naturales (Dern), del Instituto Manantlán de Ecología y Conservación para la Biodiversidad (Imecbio) de la UG.

Demetrio Meza-Rodríguez. Es ingeniero en recursos naturales. Cursa la Maestría en Manejo de Recursos Naturales en el Departamento de Recursos Naturales (Dern), Instituto Manantlán de Ecología y Conservación para la Biodiversidad (Imecbio) de la UG. Sus líneas de investigación son: manejo de cuencas hídricas y sistemas de información geográfica.

Humberto Rafael Bustos-Santana. Es ingeniero en recursos naturales. Cursa la Maestría en Ciencias en Manejo de Recursos Naturales en el Departamento de Recursos Naturales (Dern), Instituto Manantlán de Ecología y Conservación para la Biodiversidad (Imecbio), de la UG. Ha trabajado las siguientes líneas de investigación: entomología con enfoque en las familias Scarabaeidae y Melolonthidae, aspectos sociológicos del manejo de los recursos naturales e influencia de factores abióticos sobre la distribución de la vegetación. Sus publicaciones más relevantes son: "Abundancia estacional de los coleópteros nocturnos de la familia Melolonthidae (Insecta: Lamelicornia), asociados a un bosque de pino-encino 
en la Sierra de Quila, Jalisco, México”, en G. Aragón, M. A. Morón y A. Marín J. (eds.), Estudio sobre coleópteros del suelo en América, Benemérita Universidad Autónoma de Puebla, México, pp. 137-148 (2003); "Hacia una conservación basada en la gente: la Sierra de Manantlán. El análisis ecosociológico como herramienta para lograr una protección participativa de la naturaleza”, Ecología Política, 21, Madrid, pp. 43-49 (2001); "De-Constructing Conservation Flagships: The Case of Milpilla (Zea diploperennis) in the Sierra de Manantlán Biosphere Reserve in Western México", Honey Bee, 11-12 (4-1), pp. 30-31 (2001). 